\title{
Whole exome sequencing diagnosis of inborn errors of metabolism and other disorders in United Arab Emirates
}

\author{
Aisha Al-Shamsi', Jozef L. Hertecant ${ }^{1}$, Abdul-Kader Souid $^{2}$ and Fatma A. Al-Jasmi ${ }^{*}$
}

\begin{abstract}
Background: This study reports on the use of whole exome sequencing (WES) to diagnose children with inborn errors of metabolism and other disorders in United Arab Emirates.

Methods: From January 2012 to December 2014, 85 patients (46 \% females) were seen in the metabolic center at Tawam Hospital (Abu Dhabi) and WES testing was requested because definitive diagnoses were not reached by conventional methods.

Results: Eighty (93\%) patients were $<18$ years old and 44 (52\%) were $<5$ years. Sixty-eight (80 \%) patients had neurologic abnormalities. WES facilitated rapid diagnosis in $50 \%$ of the patients, especially those with mitochondrial disorders. Yet, in most cases extensive investigation was required after the results were available. Most patients with confirmed molecular diagnoses had autosomal recessive disorders and were homozygous for the rare alleles. Most patients with autosomal dominant disorders and all patients with X-linked disorders had de novo mutations. WES results were negative (no pathogenic variants related to patient phenotype were identified) in six patients and incorrect in two patients. One patient had a reported "deleterious" hemizygous mutation in SLC35A2, c.617_620del (p.Q206fs), suggesting 'congenital disorder of glycosylation, TYPE IIm', but glycosylation studies were normal and healthy brothers had the same mutation. Another patient had "pathogenic" mutation in MCCC2, c.1015G > A (p.V339M), but urine organic acids was normal. WES confirmed inborn errors of metabolism (five mitochondrial diseases, three lysosomal storage diseases, and six other disorders) in 14 patients and genetic disorders (14 neurological diseases and three non-neurological diseases) in 17 patients. Variants of unknown significance were identified in 48 patients; 12 had "confirmed pathologic variants"and 12 had "likely pathologic variants", based on consistent phenotypes, biochemical/ segregation studies, or reported pathogenicity. In 24 patients, the variants were inconsistent with phenotypes or biochemical/ familial studies.
\end{abstract}

Conclusions: Although WES provided molecular diagnoses, the results required careful interpretations and many patients required additional investigations. This tool is useful when conventional diagnostic methods fail. Staff competence in obtaining consent/ permission, interpreting the findings, and providing the proper counseling are essential before incorporating this technology into routine clinical practices.

Keywords: Whole exome sequencing, Mutations, Variants, Inborn errors of metabolism, UAE

\footnotetext{
*Correspondence: aljasmif@uaeu.ac.ae

2Department of Pediatrics, United Arab Emirates University, P.O. Box 17666,

Al-Ain, UAE

Full list of author information is available at the end of the article
} 


\section{Background}

Emirati people (citizens of United Arab Emirates, UAE) have diverse ethnicities that include lineages from the Arabian Peninsula, Persia, Baluchistan, and East Africa. The culture is primarily tribal and encourages intratribal (consanguineous) marriages [1]. Thus, "founder" mutations are prevalent, which markedly increase the frequency of autosomal recessive disorders [2]. Consistently, the prevalence of inborn errors of metabolism (IEM) in the region is high (about 1 in 1,329) [1]. Genetic disorders are also exceptionally common [3].

Diagnosing IEM is often challenging due to their genetic heterogeneity and atypical or overlapping phenotypes. Using clinical assessments and conventional investigations (having an estimated cost of about $\$ 25,000$ per patient), the rate of molecular diagnosis in these patients is usually less than $50 \%$ [4]. In these families, the lack of a diagnosis poses significant consequences, such as patients missing treatments and failure by medical staff to provide proper counseling and guidance.

The relatively new term "exome" refers to all exons in the human genome. The term "exons" refers to the $\sim 180,000$ genomic sequences, which transcribe and remain in the mature RNA. Exome constitutes only $3 \%$ of the human genome and represents the $\sim 22,000$ protein-coding genes, mutations of which are responsible for about $85 \%$ of our significant clinical diseases [5-7]. Therefore, sequencing of the exome is thought to be an efficient method of analyzing a patient's DNA to discover the genetic cause of disease. WES is especially efficient in detecting rare mutations in autosomal recessive diseases in consanguineous families.

The recently introduced WES technology offers an adjunctive analytical tool to locus-specific and gene-panel testing for patients with wide-spectrum phenotypes. This study describes diagnostic rate, advantages, and limitations of WES in the UAE.

\section{Methods}

This study was approved by Al-Ain Medical Human Research Ethics Committee (protocol number 10/09). Between January 2012 and December 2014, at Tawam Hospital (Al Ain, Abu Dhabi), 85 WES studies were requested. The risks and benefits of the test were explained to families and patients and written informed consent/ permission was obtained. WES was requested because of atypical phenotypes, overlapping features, or negative biochemical studies.

Blood (5 mL in EDTA-Vacutainer) was obtained from patients, parents and affected family members (when applicable). Extracted DNA was shipped to Whole Genome Laboratory (WGL) of Baylor College of Medicine (Houston, TX, USA). Single WES was performed at WGL as previously reported [6]. Briefly, genomic DNA from patients was fragmented by sonication. The fragments were ligated to illumina multiplexing paired-end adapters, amplified by polymerase chain-reaction assay, and hybridized to biotin-labeled VCRome (at $47{ }^{\circ} \mathrm{C}$ for 64-72 h). Paired-end sequencing was performed on IlluminaHiSeq 2000 platform. The mean coverage of the exome was $100-120 \mathrm{X}$; and $95 \%$ of the exome was covered at $>20 \mathrm{X}$ coverage. The output data from IlluminaHiSeqwere converted from bcl file to FastQ file by Illumina Consensus Assessment of Sequence and Variation Software and mapped by Burrows-Wheeler Aligner (BWA) program. Variants were filtered using stringencies of minor allele frequencies and mutation databases and disease specific databases. The results (with disease phenotype) were interpreted by molecular laboratory and medical directors certified by American board of Medical Genetics. Variant annotation and filtering were performed using Atlas-SNP and Atlas-indel.16, in addition to CASSANDRA software. Variants with a minor allele frequency of less than $5 \%$ (according to either the 1000 Genomes Project18 or the ESP5400 data of the National Heart, Lung, and Blood Institute GO Exome Sequencing Project [http://evs.gs.washington.edu/ EVS]) were retained. Variants were interpreted according to American College of Medical Genetics guidelines and patient phenotypes. Variants related to patient phenotypes were confirmed by Sanger sequencing for the patients, parents and affected relative if applicable. The interpretation of genes related to the patient's clinical phenotype was based on the clinical information of the patient. WES reports included also In silico-predictions for non-synonymous (missense) changes by SIFT and PolyPhen-2 [6]. Table 1 lists variants of unknown significance that confirmed the clinical disorder after further investigations. Tables 2, 3 list pathogenic mutations that directly confirmed the clinical disorder without a need for further investigation.

\section{Results}

Eighty-five patients (46\% females) had WES testing. WES results were divided into the following categories: [1] Variants of unknown significance, which were confirmed pathogenic (12 patients, Group A) or likely pathogenic (12 patients, Group B) based on consistent phenotypes, biochemical findings, familial (segregation) studies, or reported pathogenicity (Table 1); [2] WES diagnosis of IEM (14 patients, Table 2); [3] WES diagnosis of genetic diseases (17 patients, Table 3); [4] Variants of unknown significance, which were inconsistent with patients' phenotypes or biochemical/ familial (segregation) studies (24 patients); and [5] No pathogenic variants related to patient phenotype were identified (six patients).

Patients who had variants of unknown significance were investigated using biochemical tests, familial 
Table 1 Variants of unknown significance, which were confirmed pathogenic or likely pathogenic

\begin{tabular}{|c|c|c|c|}
\hline \multicolumn{4}{|c|}{ Group A: Confirmed pathogenic $(n=12)^{a}$} \\
\hline Genes (Isoforms) & Variants (PMID) & Diagnoses (MIM) & Comments \\
\hline \multicolumn{4}{|c|}{ Autosomal Recessive Inheritance } \\
\hline TALDO1 (NM_006755) & c.574C > T (p.A192C) (23315216) & Transaldolase deficiency (606003) & $\begin{array}{l}\text { Elevated level of polyol in urine. Affected family members had } \\
\text { the same variant. }\end{array}$ \\
\hline ETHE1 (NM_014297) & $c .488 G>A(p . R 163 Q){ }^{b}(14732903)$ & EE (602473) & $\begin{array}{l}\text { Urine organic acid and acylglycine profiles were consistent with } \\
\text { ethylmalonic encepathopathy. }\end{array}$ \\
\hline$F B X L 4 C$ & $c .1304 G>T(p . R 435 L){ }^{b}(23993193)$ & MTDPS13(615471) & $\begin{array}{l}\text { Affected sibling had the same variant and healthy siblings were } \\
\text { negative. }\end{array}$ \\
\hline DGUOK (NM_080916) & c.763_765del (p.D255del) ${ }^{b}$ & MTDPS3 (251880) & Affected sibling had the same variant. \\
\hline PEX16 (NM_004813) & $c .859 \mathrm{C}>\mathrm{T}(\mathrm{p} . \mathrm{R} 287 \mathrm{C})^{\mathrm{b}}$ & PBD8B (614877) & $\begin{array}{l}\text { Elevated level of very long chain fatty acids, low C26 beta-oxidation } \\
\text { in plasma and fibroblasts. Fibroblasts showed enlarged peroxisomes. } \\
\text { Affected sibling had the same variant. }\end{array}$ \\
\hline HEXB (NM_000521) & $c .272 G>C(p . C 91 S)^{b}$ & Sandhoff disease (268800) & $\begin{array}{l}\text { Low serum hexosaminidase activity. A pathologic variant in MCCC2 } \\
\text { [c.1015G > A (p.V339M)] was also found, but urine organic acids } \\
\text { were normal (PMID: 11181649). }\end{array}$ \\
\hline ATP8B1 (NM_005603) & c.379C $>$ G (p.L127V) b & BRIC (243300) & $\begin{array}{l}\text { Affected sibling had the same variant and healthy siblings were } \\
\text { negative. }\end{array}$ \\
\hline MTPAP (NM_018109) & c.1468G > T (p.V490L) & SPAX4 (613672) & $\begin{array}{l}\text { Developmental delay and regression at } 8 \text { months of age, central } \\
\text { hypotonia, short stature, failure to thrive, cerebellar atrophy, } \\
\text { absence-like episodes, and hip dislocation. Affected sibling had } \\
\text { the same variant. Parents were heterozygous. }\end{array}$ \\
\hline RNASEH2C (NM_032193) & c.205C>T (p.R69W) (16845400) & AGS3 (610329) & $\begin{array}{l}\text { Global developmental delay, central hypotonia, peripheral } \\
\text { hypertonia, opisthotonus, microcephaly, failure to thrive, diffuse } \\
\text { white matter hyperintensity, cortical brain atrophy, and dilated } \\
\text { ventricles. WES also reported homozygous variant in CDK5RAP2 } \\
\text { [c. } 412 \mathrm{G}>\mathrm{A} \text { (p.G138S); an unaffected sibling was homozygous } \\
\text { for the same variant] and pathogenic heterozygous mutation } \\
\text { in BUB1B [c.2441G > A (p.R814H)]. }\end{array}$ \\
\hline \multicolumn{4}{|c|}{ Autosomal Dominant Inheritance } \\
\hline FOXG1 & $c .1397 G>A(p . G 466 E)^{b}$ & Rett (613454) & Developmental regression, hypotonia, failure to thrive. \\
\hline ANK2 (NM_001148) & $C .1135 C>T(p . R 379 C)$ & Long QT (600919) & $\begin{array}{l}\text { Two siblings died of cardiac arrest; affected sibling had the } \\
\text { same variant. Parents were heterozygous, but their phenotype } \\
\text { was not investigated. }\end{array}$ \\
\hline \multicolumn{4}{|l|}{ X-Linked Inheritance } \\
\hline CDKL5 (NM_003159) & $c .593 G=/>A(p . G 198 D)$ & EIEE2 (300672) & $\begin{array}{l}\text { Global developmental delay and regression, intellectual disability, } \\
\text { hypertonia, and seizure disorder. Parents were negative. }\end{array}$ \\
\hline
\end{tabular}


Table 1 Variants of unknown significance, which were confirmed pathogenic or likely pathogenic (Continued)

Group B: Likely pathogenic $(n=12)$

Autosomal Recessive Inheritance

SLC4A4 (NM_000334)

G6PC (NM 000151)

MPDZ (NM_003829)

TTN (NM_133378)

MIPEP (NM_005932)

ALG9 (NM_024740)

c.2230G > A (p.A744T)

c.352G > C (p.A118P) (24980439)

c.[394G $>A] ;[1744 C>G]$

p. [G132S); [L582V]

c.[9160 G > C;68120 A > G]; [74633C >

T] p.[E3054;Y22707C];[A24878V]

c.1027A > G (p.K343E)

c.694G > C (p.A232P)

c.1214G > A (p.R405Q)

GLRA1 (NM_000171)

PLA2G6 (UC003aux.1)

c.154G > A (p.V52M)

Autosomal Dominant Inheritance

ABCB6 (NM_005689)

c. $[4 G>A ; 904 C>G]$ p. [V2M;L302V]

$3758 T>A(p .11253 N)$

c.865G > A (p.V289M) (8872460)
HKPX1 (149400)

NBIA2B (610217)

RTA, proximal (604278)

GSD1A (232200)

HYC2 (615219)

CMD1G $(604145$

MIPEP (602241)

CDG1L (608776)

MCOPCB7 (614497)

SCA15 (606658)

CMS2A (616313)
Severe RTA and ocular hypertension. Parents were heterozygous.

Hypoglycemia, hepatosplenomegaly, $\uparrow$ lactate, left ventricular hypertrophy, and family history of cardiomyopathy

Communicating hydrocephaly.

Mother was asymptomatic with the heterozygous c.9160 G > C and c.68120 A > G. Father was heterozygous for c.74633C > T.

Developmental delay, hypotonia, dysmorphism, microcephaly, vision loss, and atrial septum defect.

Patient had global developmental delay and regression, central and peripheral hypertonia, seizures, macrocephaly, brain atrophy, thin corpus callosum, and cysts in the white matter. WES also reported homozygous variants in NDUFV3 [c.552dup (p.L185fs)] and in KANK1 [c.1079G > A (p.S360N)].

Global developmental delay, hypotonia then hypertonia, seizures, repetitive hand movements, startle reflex to tactile and sound, dysmorphic features (elongated face, big prominent ears), tall habitus, squint, scoliosis, and precious puberty. WES also reported homozygous variants in TTC37 [c.1828A > G (p.S610G)] and in CHD8 [c.1952G > A (p.R651Q)].

Developmental regression, hypertonia, failure to thrive, scoliosis, and skin anomalies. Brain MRI findings were consistent with NBIA2B. Cousin with spina bifida and hydrocephalus. WES also reported homozygous variant in ACOX1 [c.1165A > G (p.11389V)] and compound heterozygous variant in SACS [c.2143C > A (p.P715T) and c.5732C > A (p.T1911M)].

Autism, subtle microphthalmia, and repetitive hand movements. WES also identified a heterozygous variant in CHD7 [C.5689G > A (p.E1897K)]. (CHARGE, MIM: 214800) Father had small eyes and was heterozygous for both changes.

Ataxia and cerebellar atrophy without deafness. WES also reported a variant in MYH14 [c.1126G > T (p.G376C)]. (PMID: 15015131, MIM: 600652)

Delayed motor milestone, hypotonia, pulmonary hypertension, seizures, and autistic features. Nerve conductive study was normal. Father had paranoid schizophrenia. This variant was previously reported (PMID:8872460) 
Table 1 Variants of unknown significance, which were confirmed pathogenic or likely pathogenic (Continued)

\begin{tabular}{|c|c|c|c|}
\hline KIF5C (NM_004522) & $c .404 A>G(p . Y 135 C)$ & $\begin{array}{l}\text { Cortical dysplasia, complex, with } \\
\text { other brain malformations } 2 \text { (615282) }\end{array}$ & $\begin{array}{l}\text { The disease is autosomal dominant. All family members were } \\
\text { negative for the variant. WES also reported a variant in NRXN1 } \\
\text { [c.1835A > G (p.D612G)]. Parents and one sibling were } \\
\text { heterozygous (MIM: 614325). }\end{array}$ \\
\hline
\end{tabular}

a Group A was based on consistent phenotypes, biochemical findings, familial (segregation) studies, or reported pathogenicity

bovel mutations

Mutations in bold were de novo

All mutations were homozygous, except those in Italics which were heterozygous

cWES was do in Nemozygous,

PMID PubMed Identifier, MIM Mendelian Inheritance in Man, EE encephalopathy, ethylmalonic, MTDPS13 mitochondrial DNA depletion syndrome 13 (encephalomyopathic type), MTDPS3 mitochondrial DNA depletion syndrome 3 (hepatocerebral type), PBD8B peroxisome biogenesis disorder 8B, BRIC cholestasis, benign recurrent intrahepatic, SPAX4 spastic ataxia 4, autosomal recessive, AGS3 aicardi-goutieres syndrome 3, Long QT cardiac arrhythmia, ankyrin-B-related, EIEE2 epileptic encephalopathy, early infantile

a Group B was based on consistent phenotypes or previously reported probable pathogenicity

All mutations were homozygous, except those in Italics which were heterozygous

RTA, renal tubular acidosis, GSD1A glycogen storage disease la, HYC2 hydrocephalus, nonsyndromic, autosomal recessive 2, CRS3 craniosynostosis 3, CMD1G cardiomyopathy, dilated, 1G, MIPEP mitochondrial

intermediate peptidase, CHARGE CHARGE syndrome, CDG1L congenital disorder of glycosylation, type IL, HKPX1 hyperekplexia, hereditary 1, NBIA2B neurodegeneration with brain iron accumulation 2b, MCOPCB7

microphthalmia, isolated, with coloboma 7, SCA15 spinocerebellar ataxia 15, CMS2A myasthenic syndrome, congenital, 2a, slow-channel 
Table 2 Confirmed IEM by WES. $n=14$

\begin{tabular}{|c|c|c|c|}
\hline Gene (Isoform) & Variants (PMID) & Diagnosis (MIM) & Comments \\
\hline \multicolumn{4}{|c|}{ Mitochondrial inheritance } \\
\hline tRNA Ala & $m .5591 G>A^{b}(16476954)$ & MTTA (590000) & Failure to thrive, ptosis, myopathy, normal mitochondrial studies on muscle biopsy. \\
\hline ND5 & m.13513G > A (p.D393N) $)^{b}(12509858)$ & MTND5 (516005) & $\begin{array}{l}\text { Developmental regression, dysmorphic features, esotropia, chorioretinal atrophy, } \\
\text { seizure, left ventricular hypertrophy, renal insufficiency, brain image suggestive of } \\
\text { Leigh syndrome. }\end{array}$ \\
\hline \multicolumn{4}{|l|}{ X-Linked inheritance } \\
\hline PDHA1 (NM_000284) & $c .787 C>$ G (p.R263G) (1508605) & PDHAD (312170) & $\begin{array}{l}\text { Brain image suggestive of Leigh syndrome; low complex II (succinate dehydrogenase) } \\
\text { activity in the fibroblasts. Two heterozygous ('cis' configuration) novel missense variants, } \\
\text { c.321C > G (p.1107 M) and c.338A > (p.N113l), in SDHA were found in patient and father. }\end{array}$ \\
\hline \multicolumn{4}{|c|}{ Autosomal recessive inheritance } \\
\hline FBXL4 (NM_012160) & c.1067delG (p.G356fs) (23993194) & MTDPS13 (615471) & $\begin{array}{l}\text { Developmental regression, hypotonia, failure to thrive, microcephaly, lactic acidosis, normal } \\
\text { fibroblast mitochondrial studies. Three siblings died in infancy. }\end{array}$ \\
\hline C10orf2 (NM_021830) & c.1198C > T (p.R400C) (21364701) & MTDPS7 (271245) & $\begin{array}{l}\text { Developmental regression, hearing loss, scoliosis, reduced activities of complexes I \& IV in } \\
\text { myocytes; normal activities in fibroblasts. Two cousins with Leigh disease. }\end{array}$ \\
\hline MTHFR (NM_005957) & c.1596C > G(p.Y532) (a) & MTHFRD (236250) & $\begin{array}{l}\text { Progressive encephalopathy, seizure, cerebral venous thrombosis, gangrenous like bullous } \\
\text { formation in the leg, congenital heart disease, } \uparrow \text { homocysteine, } \downarrow \text { methionine. }\end{array}$ \\
\hline \multirow[t]{2}{*}{ PYCR2 (NM_013328) } & c.28C > T(p.Q10X) (a) & HLD10 (616420) & $\begin{array}{l}\text { Developmental regression, failure to thrive, seizure, microcephaly, severe demyelination, } \\
\text { thin corpus callosum. }\end{array}$ \\
\hline & c.796C > T(p.R266X) (a) & & $\begin{array}{l}\text { Developmental delay, hypotonia, failure to thrive, microcephaly, thin corpus callosum, } \\
\text { delayed myelination. }\end{array}$ \\
\hline HEXA (NM_000520) & C.2 $\mathrm{T}>\mathrm{C}$ & Tay-Sachs (268800) & $\begin{array}{l}\text { Developmental regression, failure to thrive, seizure disorders, dystonia, feeding difficulties } \\
\text { constipation. Diagnosis confirmed by enzyme analysis. }\end{array}$ \\
\hline HEXB (NM_000521) & c.826_829del (p.E276fs) & Sandhoff (268800) & Developmental regression, failure to thrive, feeding difficulties, seizure, and vision loss. \\
\hline SUMF1 (NM_182760) & c.691dupT (p.W231fs) c.689A > G (p.E230ZG) & MSD (272200) & $\begin{array}{l}\text { Developmental delay, seizure, hepatomegaly, delayed myelination, } \uparrow \text { urine sulfatide, } \uparrow \text { urine } \\
\text { heparan sulphate. }\end{array}$ \\
\hline UROC1 (NM_144639) & $c .855 G>A(p . W 285 X){ }^{(a)}$ & UROCD (276880) & $\begin{array}{l}\text { Intellectual disabilities, attention deficit and hyperactivity disorder, hyperextensible joints, } \\
\text { ¡imidazole propionate. }\end{array}$ \\
\hline TBX19 (NM_005149) & c.604-1G > C (a) & IAD (201400) & $\begin{array}{l}\text { Intellectual disabilities, congenital hypothyroidism, two sisters died in infancy with } \\
\text { hypoglycemia. }\end{array}$ \\
\hline HSD3B7 (NM_025193) & $c .45 \_46 d e l$ (p.G17fs) (12679481) & CBAS1 (607765) & Neonatal cholestasis, hepatosplenomegaly, hypotonia, failure to thrive. \\
\hline
\end{tabular}

(a) Novel mutation; (b) heteroplasmic mutations (56-59\%). Mutations in bold are de novo, All mutations are homozygous, except those in Italics, which are heterozygous. PMID PubMed Identifier, MIM Mendelian Inheritance in Man, MTTA transfer RNA, mitochondrial, alanine, MTND5 complex I, subunit ND5, PDHAD pyruvate dehydrogenase e1-alpha deficiency, SDHA succinate dehydrogenase complex, subunit A, flavoprotein, MTDPS13 mitochondrial DNA depletion syndrome 13 (encephalomyopathic type), MTDPS7 mitochondrial DNA depletion syndrome 7 (hepatocerebral type); MTHFRD methylenetetrahydrofolate reductase deficiency, HLD10 leukodystrophy, hypomyelinating 10, MSD multiple sulfatase deficiency, UROCD urocanase deficiency, IAD ACTH deficiency, CBAS1 bile acid synthesis defect, congenital, 1 
Table 3 Confirmed genetic diseases by WES, $n=17$

\begin{tabular}{|c|c|c|c|}
\hline Gene (Isoform) & Variants (PMID) & Diagnosis (MIM) & Comments \\
\hline \multicolumn{4}{|l|}{ X-Linked Inheritance } \\
\hline PAK3 (NM_002578) & c.1279 T>C (p.Y427H) & MRX30 (300558) & Intellectual disabilities, macrocephaly, obesity. \\
\hline \multicolumn{4}{|c|}{ Autosomal Dominant Inheritance } \\
\hline BRAF (NM_004333) & c.1914 T> G (p.D638E) (19206169) & CFC1 (115150) & $\begin{array}{l}\text { Cortical blindness, seizures, stridor, constipation } \\
\text { and developmental delay. }\end{array}$ \\
\hline DYNC1H1 (NM_001376) & c. $10973 G$ > A (p.G3658E) & MRD13 (614563) & $\begin{array}{l}\text { Developmental regression, seizure, microcephaly, } \\
\text { cataract, lissencephaly, pachygyria, grey matter } \\
\text { heterotopia, hypoplasia of the corpus callosum. }\end{array}$ \\
\hline ARID1B (NM_020732) & $c .4870 C>T(p . R 1624 X)^{(a)}$ & Coffin-Siris (135900) & Mucopolysaccharidosis suspected clinically. \\
\hline ARID1B (NM_020732) & $c .3689+1 G>C$ & Coffin-Siris (135900) & $\begin{array}{l}\text { Global developmental delay, failure to thrive, } \\
\text { acute encephalopathy with hypoglycemia and } \\
\text { metabolic acidosis. }\end{array}$ \\
\hline MYBPC3 (NM_000256) & c.776delinsTT (p.A259fs) & CMD1MM (615396) & Dilated cardiomyopathy. \\
\hline \multicolumn{4}{|c|}{ Autosomal Recessive Inheritance } \\
\hline SNX10 (NM_001199835) & C.112-1G >C & OPTB8 (615085) & $\begin{array}{l}\text { Central hypotonia, optic atrophy, osteopetrosis, } \\
\text { pulmonary hypoplasia, hyperpigmented macules. }\end{array}$ \\
\hline TRAPPC11 (NM_199053) & c.2938G > A (p.G980R) (23830518) & LGMD2S (615356) & $\begin{array}{l}\text { Developmental delay, head nodding, hypotonia, } \\
\uparrow C P K \text {, } \uparrow \text { plasma phenylalanine, normal CSF } \\
\text { neurotransmitters. Homozygous c.362 T }>C \\
\text { (p.I121T) variant in COQ9 (normal muscle } \\
\text { coenzyme Q10 activity). }\end{array}$ \\
\hline PCSK1 (NM_000439) & c.1312C > T (p.R438X) & Proprotein convertase 1/3 deficiency (600955) & Brain hemorrhage, congenital diarrhea. \\
\hline ERCC5 (NM_000123) & c.205C > T (p.R69X) $)^{(a)}$ & Cockayne (278780) & $\begin{array}{l}\text { Hypotonia, developmental delay and seizure. } \\
\text { Clinically suspected to have MLCD. }\end{array}$ \\
\hline AHI1 (NM_017651) & c.1051C > T (p.R351X) (15322546) & Joubert syndrome-3 (608629) & $\begin{array}{l}\text { Intellectual disability, hypotonia, repetitive hand } \\
\text { movements, brain atrophy. }\end{array}$ \\
\hline PRX (NM_181882) & c.1090C > T (p.R364X) (21741241) & Dejerine-Sottas (145900) & $\begin{array}{l}\text { Abnormal gait, hearing loss, loss of dexterity in } \\
\text { hands, scoliosis. }\end{array}$ \\
\hline TREX1 (NM_003629) & c.341G > A (p.R114H) (21270825) & RVCL (192315) & $\begin{array}{l}\text { Cognitive impairment, hypotonia, joint } \\
\text { contracture, glaucoma, brain atrophy, sibling } \\
\text { died with the same features. }\end{array}$ \\
\hline DOK7 (NM_173660) & c.1124_1127dup (p.A378fs) \& c.1457dup (p.A487fs) (16917026) & Myasthenia, limb-girdle (254300) & Hypotonia, myopathic changes in proximal muscles. \\
\hline ADD3 (NM_016824) & c.1100G > A (p.G367D) (23836506) & Adducin-gamma (601568) & $\begin{array}{l}\text { Developmental delay, central hypotonia and } \\
\text { peripheral spasticity, cortical brain atrophy, } \\
\text { delayed myelination of white matter. }\end{array}$ \\
\hline
\end{tabular}


Table 3 Confirmed genetic diseases by WES, $n=17$ (Continued)

\begin{tabular}{llll}
\hline RECQL4 (NM_004260) & $c .1000 G>T(p . E 334 X)^{(a)}$ & RTS (268400) & $\begin{array}{l}\text { Premature, intrauterine growth retardation, } \\
\text { dry skin, clinically suspected to have MOPD2. }\end{array}$ \\
IKBKB (NM_001556) & $c .849 G>$ A (p.W283X) ${ }^{(a)}$ & IMD15 (615592) & Failure to thrive, recurrent infections, two sibling \\
& & & died with the same presentation.
\end{tabular}

(a) Novel mutation. All mutations are homozygous, except those in Italics which are heterozygous. Variants in bold are de novo pathologic mutations. PMID,PubMed Identifier, MIM Mendelian Inheritance in Man, MRX30 mental retardation, X-linked 30, CFC1 cardiofaciocutaneous syndrome 1, MRD13 mental retardation, autosomal dominant 13, CMD1MM cardiomyopathy, dilated, 1MM; OPTB8 osteopetrosis, autosomal recessive 8; LGMD2S muscular dystrophy, limb-girdle, type 2S, MLCD microcephaly-lymphedema chorioretinal dysplasia syndrome, RVCL vasculopathy, retinal, with cerebral leukodystrophy; isolated, RTS Rothmund-Thomson syndrome, MOPD2, Microcephalic osteodysplastic primordial dwarfism, type II, IMD15 Immunodeficiency 15 
(segregation) studies, and a review of the literature. Patients with consistent phenotypes, biochemical findings, familial (segregation) studies, or reported pathogenicity were considered "confirmed pathogenic" (Table 1A). Patients with consistent phenotypes or previously reported probable pathogenicity were considered "likely pathogenic" (Table 1B). The 24 patients who had "variants of unknown significance' that were inconsistent with their phenotypes or biochemical/ familial (segregation) studies were not included in this report.

\section{A. Patients' demographics}

Eighty of the 85 (93\%) patients were younger than 18 years and 44 (52\%) were younger than 5 years. Sixtyeight $(80 \%)$ patients had neurologic abnormalities. Patients had appropriate metabolic and genetic investigations (e.g., metabolic screening, microarray analysis, skin/ muscle biopsy, biochemical studies, and DNA testing) before WES testing. For most of patients, both parents DNA were tested. The cost of WES was billed to insurance companies in the UAE.

\section{B. Molecular diagnoses}

Fourteen (16 \%) patients had IEM (Table 2), 17 (20\%) had genetic syndromes (Table 3), 12 (14 \%) had variants of unknown significance confirmed to be pathogenic (Table 1A), and 12 (14\%) had variants of unknown significance likely to be pathogenic (Table 1B). Therefore, WES information was clinically useful in 55 (65\%) patients and diagnostic in $43(50 \%)$ patients.

Forty-one $(75 \%)$ of the 55 patients had consanguineous families. All patients with autosomal recessive diseases had consanguineous families (Tables 1-3); except the patient with MTHFR mutation and the patient with TBX19 mutation (Table 2) who had families of the same tribe/ region; the patient with compound heterozygous mutations in MPDZ (Table 1) and the patients with PCSK1, AHI1, and DOCK7 mutations (Table 3). The patients with autosomal dominant mutation in CHRNB1 and FOXG1 (Table 1), the patient with mitochondrial mutation in $t R N A$ Ala (Table 2), and the patient with $\mathrm{X}$-linked mutation in PDHA1 (Table 2) had nonconsanguineous families. In Table 3 , the patients with $\mathrm{X}$-linked or autosomal dominant conditions had nonconsanguineous families, except for the patient with MYBPC3 mutations.

One hundred twenty-eight variants were identified. Forty-four $(34 \%)$ variants were pathologic. Twenty (15\%) of these variants were previously reported and 24 (19\%) were novel.

\section{Autosomal dominant mutations}

Eleven (13\%) patients had autosomal dominant disorders. Five patients had truncating or missense mutations which were previously reported. Three of these mutations were de novo (Table 3). Six patients had autosomal dominant (Table 1); three were de novo variant, one was inherited from symptomatic parent, and two were inherited from parents of unknown phenotype.

\section{Autosomal recessive mutations}

Forty (47\%) patients had autosomal recessive diseases. Thirty-five patients had homozygous variants and five patients had compound heterozygous variants. Eleven pathogenic mutations involved inborn errors of metabolism (Table 2). Five of these mutations were novel, including a compound heterozygous mutation (Table 2). Eleven pathogenic homozygous mutations involved genetic syndromes (Table 3). Five of these mutations were previously reported and six were novel (Table 3).

Of the reported variants of unknown significance, nine were confirmed pathogenic homozygous mutations on further testing, six of which were novel (Table 1A). Seven of these disorders were IEM (Table 1A). In addition, six homozygous and three compound heterozygous variants were likely pathogenic; only one of these variants was previously reported (Table 1B).

\section{E. X-linked mutations}

The three patients with X-linked disorders had de novo mutations; one had pyruvate dehydrogenase e1-alpha deficiency (Table 2) and one had mental retardation, Xlinked 30 (Table 3). The patient with epileptic encephalopathy, early infantile had mosaic de novo variant (Table 1A).

\section{F. Mitochondrial mutations}

Two female patients had known pathogenic mutations. The patient with Leigh disease had $56 \%$ heteroplasmic m.13513G > A (p. D393N, ND5) mutation in the mitochondrial ND5 gene. The second patient had mitochondrial myopathy with 59 \% heteroplasmic m.5591G > A (tRNAAla), Table 2.

\section{G. Carrier status}

Carrier status for Mendelian recessive disorders was detected in nine patients. Two patients had sickle cell trait, two had $\beta$-thalassemia trait, one had CFTR gene mutation (cystic fibrosis heterozygosity), and four females had G6PD gene mutations (glucose-6-phosphatase dehydrogenase heterozygosity).

\section{H. Medically actionable genetic variants}

Medically actionable genetic variants unrelated to the clinical phenotype were identified in eight patients. Seven patients had mutations in the G6PD gene (c.563C $>\mathrm{T}$ in five patients, c.1003G $>\mathrm{A}$ in one patient, andc. $634 \mathrm{~A}>\mathrm{G}$ in one patient) and one had mutation in 
the $M Y B P C 3$ gene $(c .2148+1 \mathrm{G}>\mathrm{A})$ that causes dilated cardiomyopathy.

\section{Discussion}

In this study, WES provided useful clinical information in most patients in whom several other tests were not revealing. It attained a diagnostic rate of $50 \%$ compared with $25-42 \%$ in other studies [6-11]. This higher yield is a result of the frequent autosomal recessive diseases (mostly homozygous mutations) in the region and the multiple affected children in the same family.

Thirty-one of the $43(72 \%)$ patients with confirmed molecular diagnoses had autosomal recessive disorders compared with $34 \%$ in another study [8]. Twenty-nine of these patients were homozygous for the rare allele, indicating consanguinity played a major role in making autosomal recessive disorders frequent in this culture.

Of the patients with autosomal dominant disorders, $66 \%$ had de novo mutations (Table 1 and Table 3). In addition, all patients with X-linked disorders had de novo mutations (Table $1 \mathrm{~A}$ and Tables 2, 3). WES detected a low-level mosaicism in one patient with $\mathrm{X}$ linked de novo variant in CDKL5, which was consistent with his phenotype (Table 1). WES identified a mutation in G6PC causing GSD1a (Table 1) in a patient who was clinically suspected to have GSD III or IX due to cardiomyopathy and hepatic manifestations but had normal leukocyte enzymes for GSD III and IX. Establishing GSD1a diagnosis in this patient enabled appropriate counseling and genetic screening of the family members.

WES was especially helpful in a few patients with suspected mitochondrial disorders in whom extensive mitochondrial work-up was not revealing. Mutations in FBXL4 and C10orf2 causing mitochondrial DNA depletion syndrome were identified (Table 2). WES facilitated a rapid diagnosis in these patients with mitochondrial disorders, avoiding a need for invasive muscle or skin biopsies.

WES also provided a relatively rapid diagnosis in patients with treatable conditions, such as glycogen storage diseases (Table 1B). In addition, it provided a diagnosis in patients with atypical features of rare diseases, such as the patient with global developmental delay, repetitive hand movements, and brain atrophy without the molar tooth sign. This patient was confirmed to have Joubert syndrome due to a homozygous mutation in AHI1, c.1051C > T (p.R351X) (Table 3). In addition, WES revealed pathological variants in $A R I D 1 B$ in two patients (Table 3); one of them was suspected to have mucopolysaccridosis. Founder mutations were identified in this tribal population, such as the pathologic variant causing transaldolase deficiency (Table 1). This finding facilitated further diagnoses in our community using single gene sequencing.
It is important to note that the results of WES should be appraised carefully. In one patient, WES reported a deleterious hemizygous mutation in SLC35A2, c.617_620del (p.Q206fs), congenital disorder of glycosylation, TYPE IIm (CDG2M). However, glycosylation screening was normal in this patient and three healthy brothers had the same mutation (not listed in the table). In another patient, WES reported a pathogenic mutation in $M C C C 2$, c.1015G > A (p.V339M), but urine organic acids were normal and the patient had Sandhoff disease (confirmed by enzyme studies), Table 1.

In most cases, extensive and expensive investigations (including biochemical, genetic, and segregation studies) were necessary after the results of WES to determine the pathogenicity of the variants. In UAE, most families are consanguineous and have multiple affected individuals. Therefore, it was somewhat possible to stratify many of the variants of unknown significance as pathogenic $v s$ non-pathogenic. Nevertheless, challenges remain in the interpretation of the 12 likely pathogenic variants (Table 1b). The availabilities of functional studies, improved knowledge of rare allele frequencies of healthy individuals, and more routine use of WES will improve the yield of this powerful analytical tool.

Competent staff is needed to explain WES results and to counsel patients and their families. The metabolic centers should develop and adhere to appropriate guidelines for utilizing WES as a diagnostic tool. Position statements and best practice guidelines are available from the American college of Medical Genetics, Canadian college of Medical Genetics and European society of Human Genetics [12-14].

Many variants are confined to ethnic populations and are, thus, difficult to diagnose by conventional genetic methods $[2,15]$. The cost of diagnosing genetic disorders using conventional testing was estimated to be about $\$ 25,000$ per patient [4]. However, it is unknown whether WES would increase or decrease this cost. In this study, the cost of WES was covered by local insurance companies for UAE citizens; the cost is a critical issue for the majority of patients who are not citizens of this country.

\section{Conclusion}

In this study, with the majority of patients had consanguineous families, WES identified pathogenic mutations in $50 \%$ of cases. WES also provided population-specific information, which should facilitate molecular diagnosis of metabolic and genetic disorders in the region. However, further studies, including cost-effective assessments are needed before routine application of this test in IEM and genetic clinics. 


\section{Abbreviations}

BWA, burrows-wheeler aligner; CDG2M, congenital disorder of glycosylation, TYPE IIm; CFTR, cystic fibrosis transmembrane conductance regulator; DNA, deoxyribonucleic acid; EDTA, ethylenediaminetetraacetic acid; G6PD, glucose6-phosphatase dehydrogenase; GSD1A, glycogen storage disease la; IEM, inborn errors of Metabolism; MIM, mendelian Inheritance in man; RNA, ribonucleic acid; SNP, single nucleotide polymorphism; TX, Texas; UAE, United Arab Emirates; USA, United States of America; WES, whole exome sequencing; WGL, whole genome laboratory

\section{Acknowledgments}

We are grateful to the patients, families, and metabolic staff for their contributions to this work.

\section{Funding}

AS was supported by UAE University Biochemical Genetics Fellowship and the study was supported by College of Medicine and Health Science, UAE University grant number 31 M200.

\section{Availability of data and materials}

Not applicable.

\section{Authors' contributions}

AS, JH, AKS, and FJ collected and interpreted the data and drafted the manuscript. All authors read and approved the final manuscript.

\section{Competing interests}

The authors declare that they have no competing interests.

\section{Consent for publication}

Not applicable.

\section{Ethics approval and consent to participate}

This study was approved by Al-Ain Medical Human Research Ethics Committee (protocol number 10/09).

\section{Author details}

'Department of Pediatrics, Tawam Hospital, Al-Ain, UAE. ${ }^{2}$ Department of Pediatrics, United Arab Emirates University, P.O. Box 17666, Al-Ain, UAE.

Received: 26 April 2016 Accepted: 22 June 2016

Published online: 08 July 2016

\section{References}

1. Al Shamsi A, Hertecant JL, Al Hamad S, Souid A-K, Al-Jasmi FA. Mutation spectrum and prevalence of inborn errors of metabolism in United Arab Emirates. Sultan Qaboos Univ Med J. 2014;14:e42-9.

2. Woods CG, Cox J, Springell K, Hampshire DJ, et al. Quantification of homozygosity in consanguineous individuals with autosomal recessive disease. Am J Hum Genet. 2006;78:889-96.

3. Al-Gazali L, Ali BR. Mutations of a country: a mutation review of single gene disorders in the United Arab Emirates (UAE). Hum Mutat. 2010;31:505-20.

4. Shashi V, McConkie-Rosell A, Rosell B, et al. The utility of the traditional medical genetics diagnostic evaluation in the context of next-generation sequencing for undiagnosed genetic disorders. Genet Med. 2014;16(2):176-82.

5. Wortmann SB, Koolen DA, Smeitink JA, van den Heuvel L, Rodenburg RJ. Whole exome sequencing of suspected mitochondrial patients in clinical practice. J Inherit Metab Dis. 2015;38:437-43.

6. Yaping Y, Muzny DM, Reid JG, et al. Clinical whole-exome sequencing for the diagnosis of Mendelian disorders. N Engl J Med. 2013;369:1502-11.

7. Fahiminiya S, Almuriekhi M, Nawaz Z, et al. Whole exome sequencing unravels disease-causing genes in consanguineous families in Qatar. Clin Genet. 2014:86:134-41.

8. Yang Y, Muzny DM, Xia F, Niu Z, Person R, et al. Molecular findings among patients referred for clinical whole-exome sequencing. JAMA. 2014;312(18) 1870-9. doi:10.1001/jama.2014.14601.

9. Lee H, Deignan JL, Dorrani N, Strom SP, Kantarci S, et al. Clinical exomesequencing for genetic identification of rare Mendelian disorders. JAMA. 2014;312(18):1880-7. doi:10.1001/jama.2014.14604.

10. Lazaridis KN, Schahl KA, Cousin MA, Babovic-Vuksanovic D, Riegert-Johnson $\mathrm{DL}$, et al. Individualized Medicine Clinic Members. Outcome of Whole
ExomeSequencing for Diagnostic Odyssey Cases of an Individualized Medicine Clinic: The Mayo Clinic Experience. Mayo Clin Proc. 2016;91(3):297-307.

11. Soden SE, Saunders CJ, Willig LK, et al. Effectiveness of exome and genome sequencing guided by acuity of illness for diagnosis ofneurodevelopmental disorders. SciTransl Med. 2014;6:265ra168.

12. Green RC, Berg JS, Grody WW, et al. ACMG recommendations for reporting of incidental findings in clinical exome and genome sequencing. Genet Med. 2013;15:565-74.

13. Boycott K, Hartley T, Adam S, et al. The clinical application of genome-wide sequencing for monogenic diseases in Canada: position statement of the Canadian College of Medical Geneticists. J Med Genet. 2015:52:431-7.

14. Van El CG, Cornel MC, Borry P, et al. Whole-genome sequencing in health care. Recommendations of the European society of human genetics. Eur Hum Genet. 2013;21:S1-5.

15. Rodriguez-Flores JL, Fakhro K, Hackett NR, et al. Exome sequencing identifies potential risk variants for Mendelian disorders at high prevalence in Qatar. Hum Mutat. 2014;35:105-16.

\section{Submit your next manuscript to BioMed Central and we will help you at every step:}

- We accept pre-submission inquiries

- Our selector tool helps you to find the most relevant journal

- We provide round the clock customer support

- Convenient online submission

- Thorough peer review

- Inclusion in PubMed and all major indexing services

- Maximum visibility for your research

Submit your manuscript at www.biomedcentral.com/submit
) Biomed Central 